\title{
Bringing EU Entrepreneurs together through Cross-border Services SPOCS - a Case Study
}

\author{
Doina Banciu, Radu Marius Boncea, Carmen Ionela Rotună, Monica Anghel \\ National Institute for Research and Development in Informatics \\ 8-10, Mareşal Averescu Blvd, \\ 011455, Bucharest 1, Romania \\ doina.banciu@ici.ro,radu@rotld.ro,carmen@ici.ro,monica.anghel@ici.ro
}

\begin{abstract}
The work presented in this paper highlights the continuous improvement that has been made in terms of economic competitiveness of the European companies, with an emphasis on Small and Medium - sized Enterprises (SME's) by enabling all businesses - national and European - to benefit from available, efficient and interoperable electronic procedures.
\end{abstract}

Keywords: Cross-border services, Large-Scale Projects, Small and Medium-Sized enterprises, Services Directive.

\section{Introduction}

In the literature review (Adshead, 2002; Hooghe and Marks, 2004; OECD, 2005) ([1], [11], [4]) governance in an EU context is defined as a dense network of policy actors involved in the policy process to carry out social functions. Increasing emphasis on the principles of good governance has been associated with a strengthened role for regions in economic development. For entrepreneurship development this is particularly important since it is at the sub-national level that entrepreneurs are most likely to come into contact with the state. Two governance issues are particularly relevant to the promotion and facilitation of successful cross-border entrepreneurship. The first relates to the suitability of the governance structures, at different levels, in place to achieve this; the second to the nature and extent to which entrepreneurs my be involved in the policy-making process. In recent years, the EU has played an important role in the establishment of governance structures at national, regional and local levels and it has also encouraged a process of administrative restructuring and enhancing institutional capacity, involving more power being delegated to the regional and local levels, as well as shaping the relations between the public and private sectors, by promoting the creation of networks and partnerships.

In this context services currently represent two-thirds of the EU's GDP and employment, only making up for around one-fifth of total intra-EU trade and only about 8\% of European SMEs do business in other Member States.
This lack of dynamism not only hampers choice for consumers, but also prevents small and innovative businesses to grow, develop their activities and become more competitive. In order to unlock this potential of the Single Market for services by 2012, the European Commission has adopted a set of targeted actions to tackle remaining problems.

The Services Directive aims precisely at removing unnecessary and burdensome obstacles to trade in services in the Single Market. One year after the implementation deadline, the Commission and the Member States have completed an assessment of how the Directive has been implemented on the ground. The results of this so-called "mutual evaluation" exercise conclude that, while much has been achieved so far, the Single Market for services is not yet delivering its full potential [17].

Given the potential for additional growth this represents, a more thriving EU services sector is a priority for the European Commission, as services are the driving force of the EU economy and statistics show that around nine out of ten new jobs are created in this sector. As identified in the Commission's Annual Growth Survey, the EU will only meet its ambitious Europe 2020 targets for sustainable and inclusive growth if urgent structural reforms are prioritized in services and product markets to improve the business environment.

Nevertheless, the EU Single Market for services is still not functioning properly and needs further attention - this is the outcome of an extensive evaluation of the Services Directive. 
A number of legal and administrative barriers still make it difficult for businesses and consumers to buy and sell services in and from other EU countries. They also make it difficult for SMEs and the self employed persons to start new activities or to expand at home or abroad. In some cases, SMEs are required to carry out burdensome time-consuming formalities and registration procedures before they can cross the border to provide a service [12].

The Service Directive adopted at the end of 2006 has been a crucial milestone in improving the legal framework for services in Europe. It aimed at removing a large number of obstacles to service trade, requiring EU Member States to remove unjustified or disproportionate legal and administrative barriers to the setting-up of a business or the provision of cross-border services in the EU. Also, it aims to dismantle barriers affecting service recipients wanting to have access to services from other Member States. The Directive had to be transposed and implemented in all EU countries by 28 December 2009. It highlights that further improvements need to be made to make EU markets work better and create the growth and jobs the EU desperately needs and it also identifies key priority actions to carry out to move towards a better functioning Single market for services.

The Directive applies to two main situations:

- The permanent establishment of businesses, i.e. cases where an individual entrepreneur or business wants to set up a permanent establishment (such as a company or a branch) in its own country or in another EU country;

- The cross-border provision of services, i.e. cases where a business already established in an EU country wants to supply services in another EU country, without setting up a permanent establishment there, or cases where a consumer living in one EU country wants to buy services from a provider established in another EU country. Examples: a painter established in France is commissioned to paint a house in Germany, or a consumer from Finland wants to use an architect established in Estonia [12].

The Services Directive obliges each EU country to set up a "Point of Single Contact" - e-government portals for businesses that are now operational in most Member States allowing businesses to complete all administrative steps online; obliging national administrations to cooperate with administrations in other EU countries to avoid duplication of controls on businesses and significantly reduce the administrative burden on business entities.

Furthermore, the Directive brought about the creation of a comprehensive network of administrative cooperation to facilitate the free movement of services, which now links up over 5000 authorities across the EU. A multilingual electronic tool, the Internal Market Information system or "IMI", is used to allow a fast and efficient exchange of information between these authorities.

It also gives consumers wider choice, better value and easier access to services across the EU: consumers will get better information on service providers and their services, such as information on after-sales guarantees and redress or the fact that consumers should suffer no discrimination based on their nationality or place of residence.

In economic terms, conservative estimates predict that the implementation of the Services Directive has the potential to bring about economic gains of up to $€ 140$ billion, representing up to 1.5\% growth of EU GDP [4].

SPOCS is a three-year large-scale pilot project launched by the EC in May 2009 which aims to significantly facilitate life and increase transparency for SMEs and consumers when they want to provide or use services in the single market. Businesses are invited to test-drive the new services using SPOCS that will support travel agents, real estate agents and master builders in establishing a presence and doing business in other EU countries (e-practice).

Run largely with and/or by Member States, the LSPs develop practical solutions tested in real government service cases across Europe. These practical solutions will ensure that government administrations of different countries in the EU can speak to each other digitally despite different national technical specificities and languages [15]. The project consortium is composed of 34 partners, from 16 member states, including Romania, comprising of national public administrations, representatives 
thereof, local authorities, industries and universities, thus covering all walks of life.

Building on compliancy with the Services Directive (CE/2006/123) , SPOCS has been set up on the basis of the 2008 CIP ICT PSP Programme (project reference: 238935). The implementation of the SD concerns a large variety of economic activities (about 70\% of EU GDP and total employment) and requires important legislative changes in all Member States (MSs) and the undertaking of a number of ambitious projects, such as setting up the national "Points of Single Contact" (PSCs) - eGovernment portals for entrepreneurs active in the service sector and citizens.

For example, if a person wishes to open or extend a business abroad he/she has to travel to the city where he/she wants to expand his/her business, go to City Hall, the Trade Register, etc.. This means a waste of time and money and a lot of bureaucracy - applying for licenses, permits and completing other administrative procedures. With SPOCS, the entrepreneur can complete the formalities from his office, online, quickly and efficiently, only by filling-in the necessary forms and loading documents in electronic format.

In order to fulfill the administrative obligations and formalities in a uniform manner, SPOCS and its contributors, reuse key building blocks from other EU projects (such as STORK Secure idenTity acrOss boRders linKed and PEPPOL - Pan-European Public Procurement OnLine) and build upon existing, interoperable standards, frameworks and national solutions to improve cross-border administrative processes.

SPOCS will, therefore, be carried out in a transparent way to facilitate consensus by public administrations, their suppliers and partners, industry and standardization bodies without replacing national systems.

The simplification measures foreseen by the SPOCS project should enhance the quality of completing electronic procedures for crossborder activities. The implemented project results will lead in reducing bureaucracy by electronic means, increasing transparency in the public sector, improving on-line services and generating benefits for global economic growth.

\section{Work Overview}

\subsection{SPOCS building blocks}

The SPOCS building blocks create the interoperable services layer necessary to exchange documents, services and information between different Service Providers (SPs) in MSs and the PSCs through which they complete their procedures.

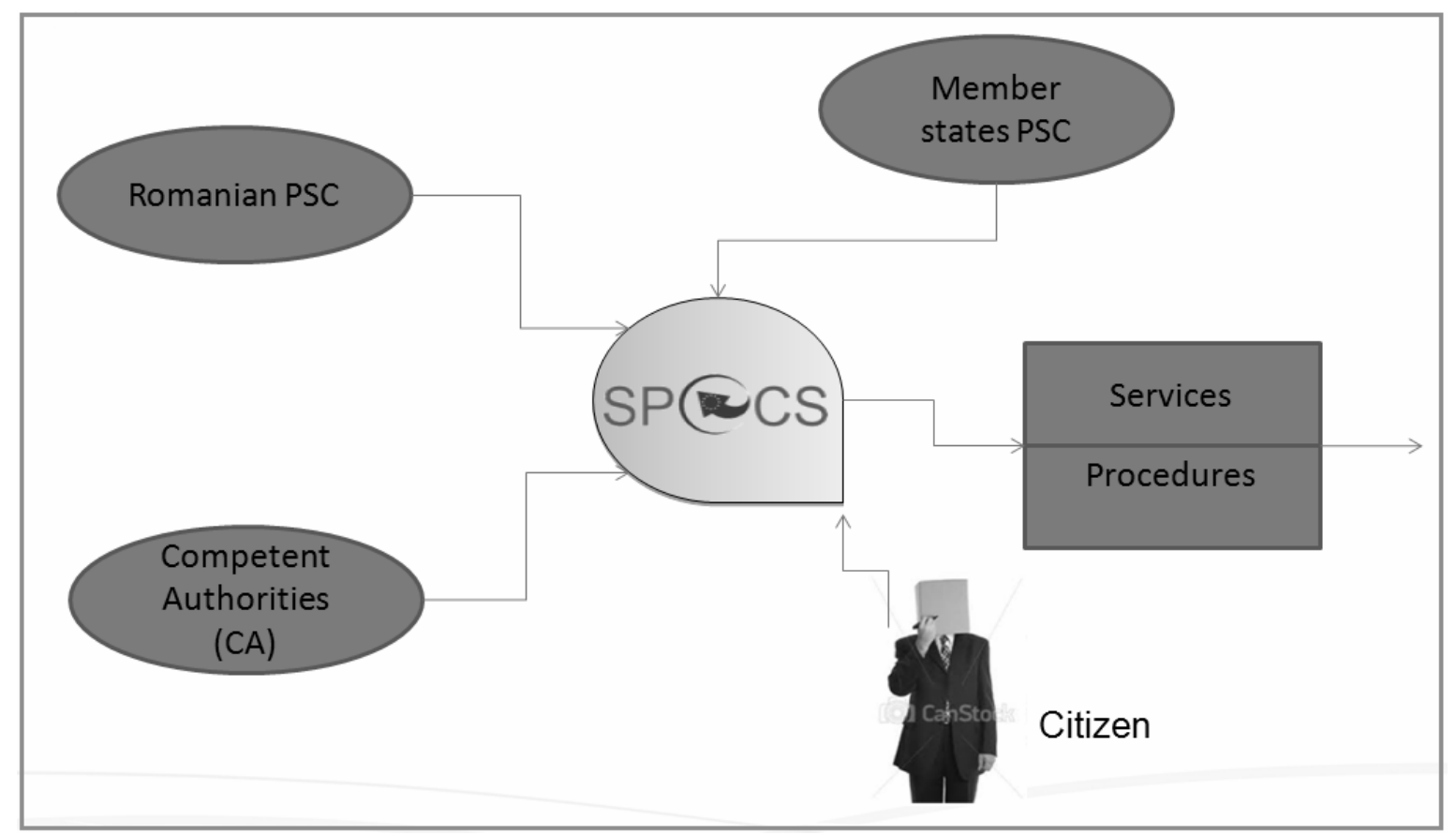

Figure 1. SPOCS Interactions and functionality 
The SPOCS pan-European interoperability layer is composed of 5 building blocks focusing on answering the following key questions related to electronic procedures in Points of Single Contacts portals:

1. Syndication: The building block, responsible for mapping of equivalent documents required by application processes in MS and supporting automatic discovery of electronic services.

2. eServices: The solution for eServices, provides search, access and delivery of information regarding the Services Directories and eServices across MS. The eServices building block aims at establishing interoperability between the
PSCs, Service Catalogues (SCs) and electronic Services Directories (eSDs). There are two main components: Meta Information Data Base (MIDB) and the Work Package 4 Interoperability Layer (WP4IL) Open Modules: Transformation, Syndication, Search, Access and Provision.

3. eDocuments: The building block, responsible for the processing and validation of electronic documents in different formats, introduces a multilayered eDocument container format: the Omnifarious Container for eDocuments (OCD). The container is able to hold any kind of electronic data in its payload layer. This ensures that every existing document can be transferred using an OCD container,

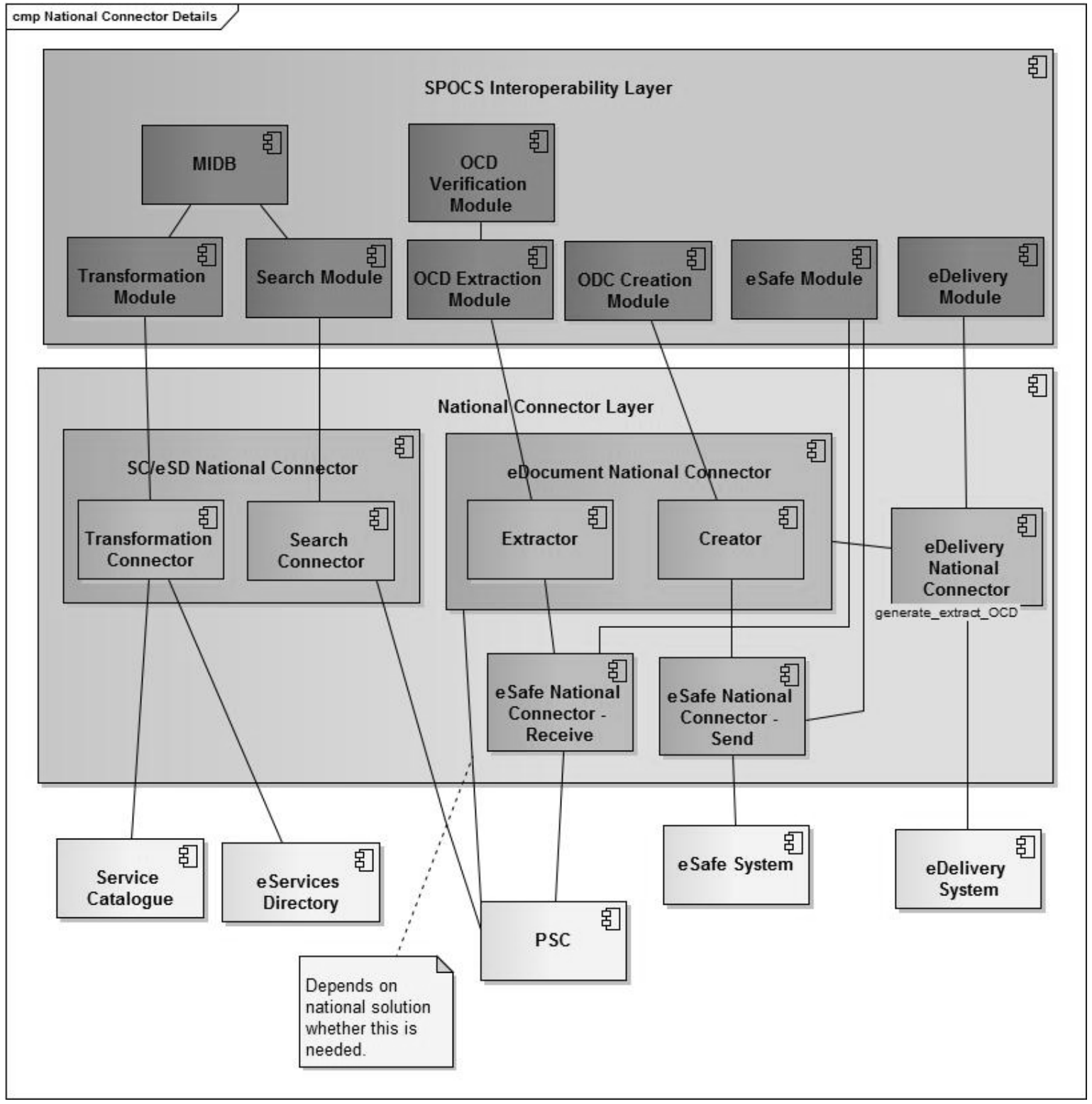

Figure 2. SPOCS Interoperability layer 
no matter if it is electronically signed or not. The OCD contains three layers: Payload Layer, Metadata Layer and Common Authentication Layer.

4. eDelivery: The SPOCS eDelivery defines a protocol to interconnect different eDelivery systems in Europe. The main functionalities are conversion between different eDelivery messages and transport protocol formats, provision of trust and evidence of end-toend delivery. This protocol is based on three key components: SPOCS Gateway, SPOCS TSL, SPOCS Evidences.

5. eSafe: The SPOCS eSafe building block integrates secure storages of documents and their access by authorized parties and specifies an integration protocol that is based on well-established standard protocols such as HTTP or Web services. Furthermore, the SPOCS eSafe integration protocol is inspired on the proven EPS epayment standard for integrating payment activities in foreign portals. The whole communication is secured by using SSL/TLS.

The technical specifications on eDocuments, Content Syndication, eDelivery, eSafe,
eService Directories have been subject to an open consultation among other stakeholders both the MS and companies providing IT solutions. For instance, commerce across borders means taking into account the most stringent business regulations of all the countries involved. And this may mean transmitting sensitive, confidential information. So when transmitting qualifying evidence to set up a company, for example, the sender needs confirmation of receipt while the receiver needs to be able to read all security information and check the data has not been tampered with.

The SPOCS transport 'envelope' can contain all this, semantically and securely, because it has taken the Virtual Company Dossier (VCD) schema from PEPPOL and developed it into the OCD for SPOCS. It is an excellent example of the way the LSP concept of building blocks is creating synergies between the different projects.

\subsection{SPOCS working packages}

The SPOCS development project has been divided into 7 major Working Packages (WP):

- WP1: Content syndication, multilingual issues and glossary - Syndication, related to

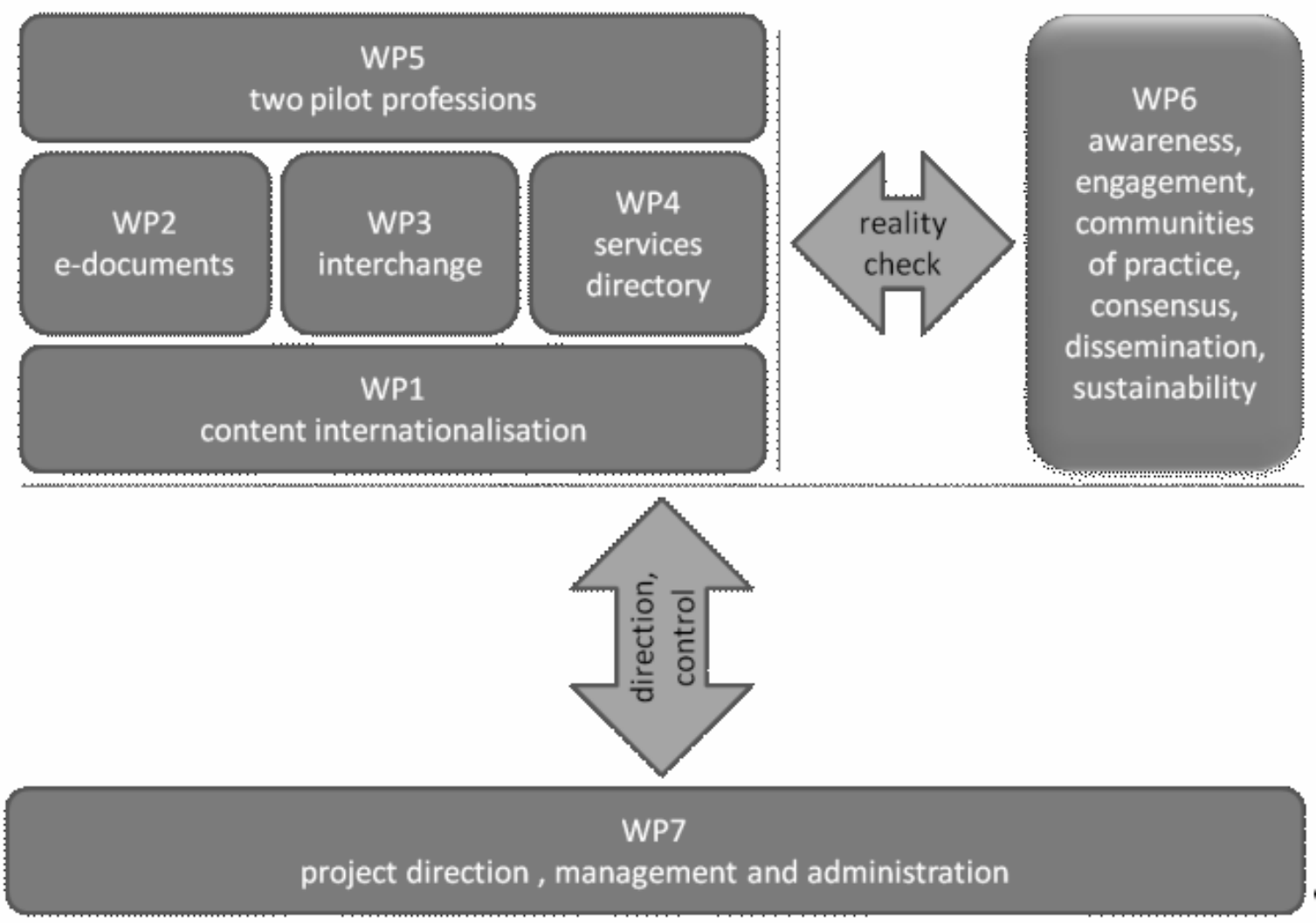

Figure 3. Working packages 
glossaries and the multilingual reality, is used to supply SPOCS enabled PSC's the metadata on the available licenses, procedures and other relevant information available from competent authorities. Cross-border content syndication's effective implementation needs common ground based on national best practices and open specifications.

- WP2: eDocuments - Official cross-border documentation needs to be automatically processed regardless of its origin or language, needs to be understood by PSCs, CA and SP and documentation's authenticity needs to be validated respectively. SPOCS develops existing interoperability models and common specifications for documents that will assist convergence and reduce heterogeneity.

- WP3: Interoperable delivery, eSafe, secure and interoperable exchanges and acknowledgement of receipt - SPOCS provides solutions such that competent authorities (CA) and PSCs (Point of Single Contact) of one MS can effectively communicate the outcome of an administrational procedure (usually eDocuments) to a service provider or agency in another Member State. eSafe makes online transactions more efficient, comfortable and user friendly foreground developed in PEPPOL (technical infrastructure \& eSignature) and STORK (eID).

- WP4: Interoperable eService Directories Definition and description of services form a better understanding and recognition of eServices that are provided in different national service directories. For the implementation of the EU Service Directive it is necessary to find the appropriate points of single contact and responsible public agencies for the processing of applications. SPOCS will focus on structuring and connecting resources and systems (i.e directory services/relational databases) containing information about authorities and services.

- WP5: Experimenting with Professions The main objective is to experiment with the provision of services related to two professions. Real cases of two professions use and customization of: the syndicated content, the eDocuments, the service provision environment (implemented/deployed operations, populated edocument safe, monitoring and time stamping functionality for the transactions, cross-border use of electronic signatures) workflows, the information systems, the legal framework, the model processes and the content of the e-Safe.

- WP6: Awareness raising, dissemination, stakeholder groups engagement and growing

- communities of practice.

- WP7: Project Management.

\section{SPOCS Work Overview in Romania}

Being actively involved in all working packages, with the technical team at ICI Bucharest working especially on WP1 ("Content syndication, multilingual issues and glossary") and WP4 ("Interoperable eService Directories"), we are now at the end of SPOCS year 3 and we are committed as much as we were in the beginning to "making business easier" in Europe.

WP1 and WP4 provide for a mechanism that establishes semantical equivalence trough the MIDB (Metadata information database) that is filled with the updated metadata from every PSC and a search mechanism that allows a PSC to search the information in another PSC so the SP knows which document is equivalent in the entrepreneurs own country and can be directed to the apropriate page and information on how to get it.

Content syndication is the set of mechanisms that distribute public information (or meta information describing this information) on eGovernment services between information systems of governmental authorities. These syndication standards enable PSCs to present the SP information on documents from his/her home country, Romania for example, that are equivalent to those required for an eGovernment service in the area of jurisdiction (country or state) of the PSC.

WP1 specifies the syndication standards and guidelines in such a way that they can be used for 
other purposes as well (for instance, exchange of information between eServices Directories).

The main objective of WP4, in which our team has been actively engaged, was to provide definitions and descriptions of services and eServices, that are provided in different national service directories. This leads to the following specific objectives:

- Establish a meta structure concerning life events, products and services in general and connected to life events;
- Identify and deliver the required specifications for interoperability between national approaches;

- Implement required modules;

- Deliver a common governance scheme / guideline for directories of public services;

- Evaluate take-up and usage of concepts and common specifications in the pilots.

Figure 4 shows the SPOCS syndication architecture as an Unified Model Language UML component diagram. It depicts two

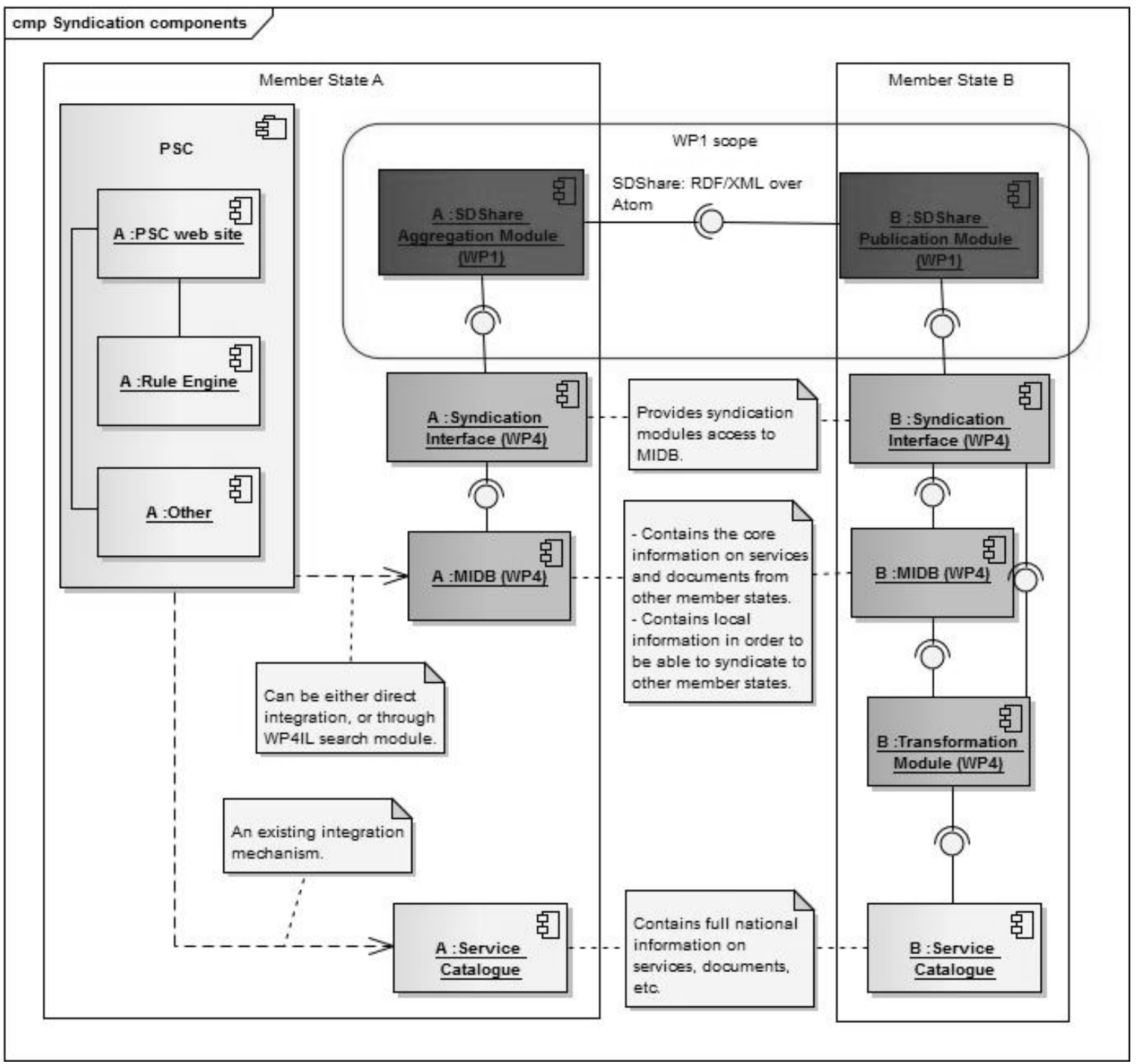

Figure 4. SPOCS Syndication Architecture

- Establish a common view on existing approaches regarding service directories and Service Oriented Architecture (SOA) approaches for eGovernment in member states and their possible solutions towards interoperability; member states: A and B. Member state B publishes information about its eGovernment services and documents. This information is consumed by member state A and used to enrich the functionality of its PSC for visitors from member state $B$. Both member states have their own national service catalogues. Using the 
SPOCS "Mapping" and "Transformation" modules provided by WP4, the core information about B's services and documents is transferred to B's MIDB. Using the "Syndication MIDB Access" module the "Syndication Publication Module" supplied by WP1 publishes the information, which is picked up by the A's "Syndication Aggregation Module". This module updates A's MIDB using A's "Syndication Interface" module.

In order to use B's information, A's PSC queries its MIDB, either via a direct interface or by using the "Search" module of WP4. The PSC can get results in the form of semantic descriptions in RDF(Resource Description Framework)/XML.

As PSCs will typically contain a rule engine that evaluates pre-defined rules based on properties of the visiting SP, and suggests which of B's services and documents are applicable to that SP. (If the rule-engine is OWL (Web Ontology Language) - capable, it will be able to use the RDF directly.) The PSC may contain other functional components.

\subsection{SPOCS environment}

Our team quickly adapted to the technical project requirements, installing and configuring a JAVA environment, using Java SDK 1.6, Java EE 1.6 and a wide range of open source platforms, frameworks and IDEs: SpringFramework and JaxWS for web services development, JENA for the semantic layer, MySQL for database storage, Eclipse-Maven M2E for IDE, Java Hibernate and Persistence API for database objects mappings and relations, Nexus for dependency packages repository, Maven for dependency management and automatization of builds, Hudson for continuous integration, JIRA for task management, JCE for implementing cryptographic extension, Semagia Atomico for syndication, Tomcat or JBoss as web servers.

All working packages JAVA API deliverables must ensure Confidentiality, Integrity, Authorization, Non-repudiation and Authentication of data, thus an extensive research is being conducted in cryptography, digital signatures, secure timestamps and audit logs.

The ICI software developing team has decided to add intelligence to the system by adopting several semantic technologies to describe the logical entities and procedures, such as: RDF and OWL to declare and use semantic entities, SparQL as a query language and SDB for the storage of the RDF entities.

The technical team in Romania adopted open standards such as $U M L$ to describe generic SPOCS entities and processes and after careful evaluation of the technical aspects concerning SPOCS, the team needed to extend the models to specific entities using similar methods with Universal Business Language - UBL, we published data to Open Data, syndicated data from authorities and Open Data, built semantic equivalences, associations, exclusions of the models based on entities, reengineered the $e$ Documents and eServices to use the semantic models and the semantic methods.

The research on technologies available and suitable adopted in SPOCS are: Advanced Electronic Signature (XMLDSIG, XAdES, XAdES - BES, XAdES-T), thus we adopted TSL (Trust-Service Status List) trust model to ensure non-repudiation, authentication and encryption of digital data, adopted TSL for data communication and storage and also added metadata support to describe entities, models and services.

\subsection{The Poi nt of Single Contact (PSC) in Romania. Implemen ting the Services Directive calls for setting up Points of Single Contact (PSC).}

From a national perspective one of the most important strategic results is the launching of an operational Romanian PSC (http://www.edirect.eguvernare.ro) that will ensure an open eGovernment for Romanian citizens, stakeholders, SME's and public administration.

The PSCs are acting as intermediaries between service providers and the national public administrations. The goal of these "one-stop shops" is essentially to fulfil the two following functions: information dissemination and case management/processing. SPOCS aims to build the next generation of PSC for business start up. Therefore in doing so, SPOCS shall contribute substantially to the efficiency and effectiveness of business start up procedures, thus limiting the administrative burden of starting up a business. Bench learning shows that the following benefits shall arise: $€ 12 \mathrm{M}$ administrative savings per annum for small countries and improving offline services and 
taking them online can save up to 5 times the costs in administrative procedures

Therefore, SPOCS is expected to provide for both savings and competitiveness a way to ease up the process of starting up a business.

July 2011 saw the launch of the first services using SPOCS that will support travel agents, real estate agents and master builders in establishing a presence and doing business in other EU countries. Businesses are invited to test-drive the new services, which will initially span 5 Member States. Austria, Germany, Greece, Italy and Poland will all demonstrate their services in the SPOCS pilot. Other EU Member States will join later in the year. The live pilots will run for at least 12 months, including extra pilots for 8 months (www.euspocs.eu/pilots/).

SPOCS will contribute to: fostering competitiveness; achieving greater interoperability; streamlining electronic procedures; gaining efficiency in administrative simplification and modernization; improving usability and attractiveness of PSCs; increasing transparency and user-friendliness of procedures for service providers and service recipients; increasing cross-border activities; stimulating cross-border cooperation between Member States; reducing misuse/ fraud. The aim of SPOCS is to develop an interoperability layer to foster the services economy in Europe by facilitating the Service Providers to apply via the Points of Single Contact for businesses the EU Member States have set up. Therefore, the aim of the SPOCS pilot is to show that the building blocks developed within SPOCS composing this interoperability layer indeed do function in a real life environment. The prospects of the SPOCS project are positive and further promising as the project is on track in developing, deploying and testing the software necessary for the running of the pilots [19].

PSCs are online e-government portals that offer information about the rules, regulations and formalities that apply to service activities and complete the administrative procedures online (by submitting the necessary application forms and supporting documents, etc. electronically). Citizens and entrepreneurs no longer have to go to the individual offices of different authorities in different countries, one by one. In each EU country, applications can now be dealt with online through one single access point, the
PSC. For example, a company based in Greece and willing to provide catering services in Italy will be able to ensure that all the administrative procedures are filled in online through the Italian PSC.

Through the PSC, connected to the central public administration and other competent authorities, service providers can carry out procedures and formalities remotely, by electronic means all procedures and formalities needed in order to access and exercise service activities. The system can technically support, administrative simplification and reform processes. In relation to citizens, the system allows stage implementation of public remote services and provides detailed information in an accessible manner.

\section{Piloting}

SPOCS went live with 5 pilots (Germany, Austria, Italy, Poland and Greece) and 3 professions ("Travel Agent”, "Real Estate Agent" and "Master Builder"), on the 1st of July 2011. The pilots are using the SPOCS building blocks for Syndication, eDocuments, eDelivery, eSafe and eServices in the national production environment of the Points of Single Contacts in the 5 Member States.

The piloting services are opened to entrepreneurs interested in providing services abroad, entrepreneurs wanting to set up a business abroad, natural persons wanting to open a branch abroad, service providers and intermediaries that wish to provide temporary services or to start up in another piloting country as "Real Estate Agents", "Travel Agents" and "Master Builders", internet users that need information on how to start a business as "Real Estate Agents" in one of the piloting countries.

On a national level the team in Romania must define the common specifications and procedures concerning the development of a pilot on the "Travel Agent" profession. This means to identify the key person from the central public administration and based on a deep analysis to design the programme specifications and elaborate it. Also, the procedures have to ensure the interoperability between the SPOCS procedures and the national PSC and between the national procedures and SPOCS procedure. 
The pilot $p$ roject will be implemented in different phases and a special emphasis will be drawn $o n$ the devel opment of common specifications and tools for ele ctronic services, su ch as technical and s emantic interoperability, the promotion of electronic documents (eDocuments) and the creation of a services directory.

\section{Conclusions}

The work achieved within SPOCS aims at elaborating common specifications, open modules and organizational solutions relying upon open standards, thus ensuring these are portable and reusable by all Member States. In particular, building blocks will be published under a license compatible with the EU Public License.

The SPOCS project will provide solutions among others in the following fields:

- Provision of information to the service provider combing information gathered by different Member States i.e. processes from a Member State related with documents of another Member State.

- Provision of ready to use and tested solutions. The products of SPOCS projects will be available for everyone without any charge. Every country will be able to implement either all or just chosen modules and provide new functionalities for the service providers on the PSCs.

- Submission of electronic documents that can be easily checked for their validity and their suitability for the specific process from different Member States.

- Automatic retrieval of documents from a source of authentic documents installed in another Member State.

- Provide a secure communication channel from the Pont of Single Contact of a Member state to the service provider of another MS with all the appropriate acknowledgment receipts that are necessary for legal results that the EU Service Directive has foreseen.

A special effort will be placed on the dissemination of open specifications, the sharing of knowledge and best practices, as well as the encouragement for further cooperation. A wide range of organizations is represented among the partners, from decision makers to service providers and IT solutions.

The results of the extensive evaluation of the Services Directive show that the Directive has been a major step towards a better functioning Single Market for services. Burdensome requirements -such as unnecessary or overcomplicated prior authorizations schemes have been abolished or simplified significantly in many service sectors. However, the evaluation has also clearly shown that the Single Market is not yet functioning to its full potential. Problems and barriers remain in particular when businesses want to provide their services temporarily across borders. This slows down growth opportunities notably for small companies and reduces choice for consumers [12].

\section{Acknowledgements}

This work has been carried out as part of the SPOCS (Simple Procedures Online for Crossborder Services) project a Large-Scale Project (LSP) launched and co-financed by the European Commission (EC) in May 2009.

\section{REFERENCES:}

1. ADSHEAD, M., Developing Euro pean Regions? Co mparative Governa nce, Policy Netwo rks a nd Euro pean Integration, Aldershot: Ashgate, 2002.

2. BANCIU, D., D. NICA, Le gouvernement elec tronique. Concepts appliques en Rou manie. Bucharest, Editura Tehnică, 2005.

3. BANCIU, D., e-Services in Futur e Internet - S tudy-case on Ro mania. 6th Ministerial Conference "Borderless eGovernment Services for Europeans", Session 15: Impact of Future Internet on eGovernment Services, November 17th 18th, 2011, Poznan International Fair, Poland.

4. BANCIU, D., Digital Cu Iture and Informing t he Citizen - Fi rst $S$ tep in Reshaping t he Economy: e -Romania Concept, Global Forum, Bucharest, October 19th - 20th, 2009.

5. BANCIU, D., e-Romania - A Web Gateway $f$ or Ci tizens' Info rmation, 
Studies in Informatics and Control, vol. 18(3), September 2009, pp. 205-210, ISSN 1220-1766.

6. BANCIU, D., "e-România" from Concept to Impl ementation, Automation Computers Applied Mathematics (ACAM) Scientific Journal, vol. 18(2), pp. 246-249, ISSN: 1221$437 \mathrm{X}$.

7. EIBL, HERWIG, KAMING, KUSTOR, LEDINGER, LEITOLD, MEDIMOREC, PIRKER, REICHSTAEDTER, RUPP, SCHEIDBACH, WAGNERLEIMBACH, Administration on the net. The ABC guide of e-Government in Au stria, Vienna, 2011, ISBN 978-3200-02352-9.

8. HOOGHE, L., G. MARKS, Unravelling the Central State, but How? Typ es of Multi-level Governa nce, American Political Science Review, vol. 97(2), 2003, pp. 233-243.

9. TSELENTIS, G., A. GALIS, A. GAVRAS, S. KRCO, V. LOTZ, E. SIMPERL, B. STILLER, T. ZAHARIDIS, (Ed.), Towards the Future Internet. Emerging trends from European re search, IOS Press, Amsterdam, Berlin, Tokyo, Washington DC, ISBN 978-1-60750-538-9 (print), ISBN 978-1-60750-539-6 (online).

10. Building Co mpetitive Regions: Strategies and Governance, OECD, Paris: OECD, 2005.
11. Expected Eco nomic Benefit $s$ of the European Se rvices Directive, Netherlands Bureau for Economic Policy Analysis (CPB), November 2007.

12. ICT fo $\mathbf{r} \mathbf{s}$ ocietal Challenges, Digital Agenda for Europe 2010-2020, European Commission, Luxemburg: Publications Office of the European Union, 2011, ISBN 978-92-79-19599-0.

13. Research.eu. Focu s Ma gazine," Build, Connect, Grow", No. 12, February 2012, ISSN 1831-1903, Published by CORDIS Unit, Publications Office of the European Union.

14. The Wo rld in 2011. ICT Facts and Figures, International Telecommunication Union - ICT Data and Statistics Division Telecommunication Development Bureau, 2011, Geneva, Switzerland.

15. User expectations o f a li fe ev ents approach for d esigning e-Government services, Final Report Summary, European Commission, Information Society and Media, ISBN 978-92-7920619-1., Luxembourg: Publications Office of the European Union.

16. http://www.eu-spocs.eu;

17. http://www.buildconnectgrow.net/;

18. http://www.edirect.e-guvernare.ro;

19. http://www.eubusiness.com;

20. http://www.epractice.eu. 
